\title{
Fuzzy Classification of ECG Signals Using A QRS-Like FIR Filter Bank with Lattice Structures
}

\author{
Jassim M. Abdul-Jabbar \\ drjssm@yahoo.com \\ Rasha Waleed Hamad \\ Rasha_Waleed87@yahoo.com \\ Department of Computer Engineering- College of Engineering-University of Mosul- Mosul- \\ Iraq.
}

\begin{abstract}
Significant features of the ECG signal include the $P$ wave, the QRS complex, and the $T$ wave. This paper focuses on the detection of the QRS complex. A $1^{\text {st }}$ order Gaussian derivative function has a similar shape to QRS complex part of the ECG. In this paper, an FIR filter bank is efficiently designed with lattice structures for QRS features extraction, using Gaussian function with standard deviation value $\sigma=4.5$. ECG features are taken after three-level decompositions of the proposed filter bank. Significant energy values of the filter bank output coefficients are calculated and treated as crucial points for identification of the diseases/disorders in the ECG signal. Such values are used for the design of a rule-based fuzzy classifier.

Keywords: ECG signal, QRS complex, 1st order Gaussian derivative function, FIR filter bank, lattice structures, fuzzy classifier.

\section{التصنيف المضبب للإشارات تخطيط القلب باستخدام جرف مرشح FIR ذو إستجابة مشابهة لشكل QRS وبهيطاكل متثنابكة}

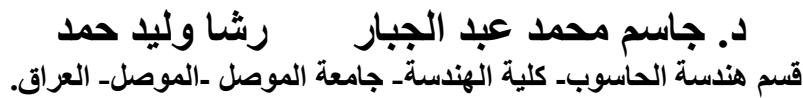

الخلاصة

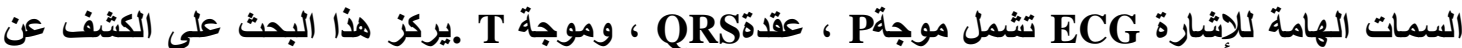

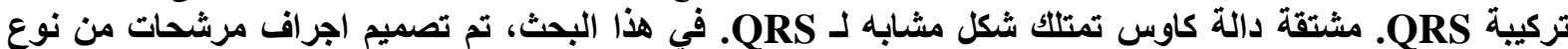

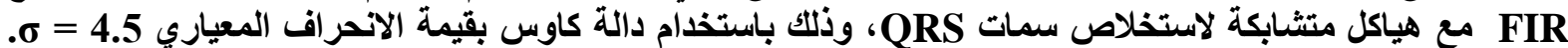

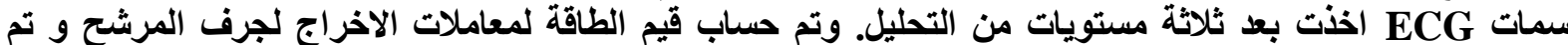
اتخاذها نقطة حاسمة لتحديد الأمراض/ الاضطرابات في إثدارة ECG. تلك القيم تم إستخدامها لتصنيم التصميم قواعد المصنف




\section{1- Introduction}

The Electrocardiogram (ECG) is a vital sign signal for heart functional investigation. This electric signal is generated from human heart to create the cardiac cycle, which generates the blood circulation. It is composed of three basic components, named P wave, QRS complex and $\mathrm{T}$ wave as shown with their intervals in Figure 1. $\mathrm{P}$ wave is generated during atrium depolarization. After that, QRS complex is generated during ventricular depolarization and T wave is generated when ventricle recovery occurs.

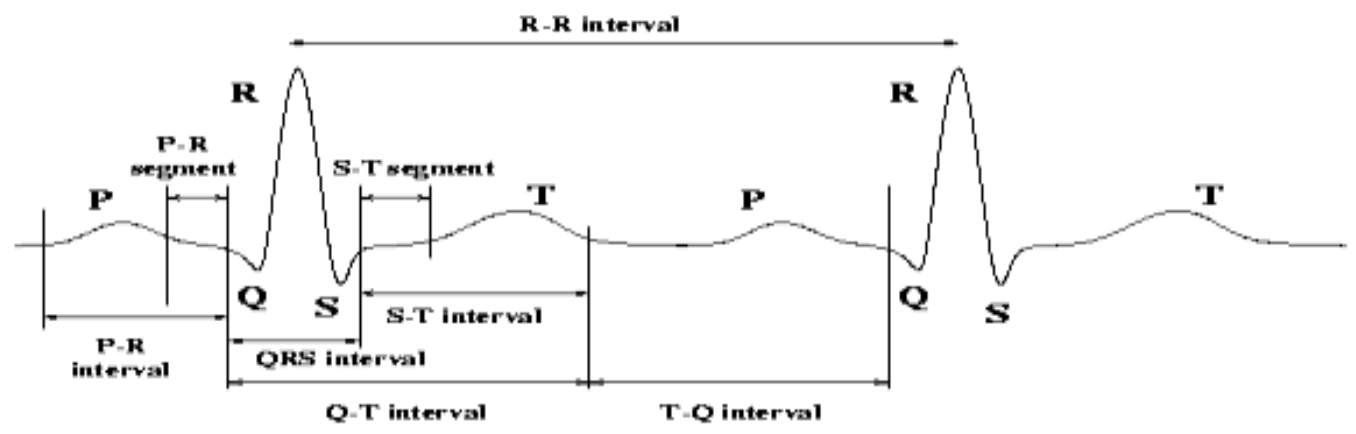

Fig.(1): Definition of waves, segments and intervals in the normal ECG waveform, [1].

This ECG is used clinically in diagnosing various abnormalities and conditions associated with the heart. The R-R interval is the distance between two subsequent QRS complexes and represents the heart rate (HR). Normal HR is between 60 to 100 beats per minute (bpm). A high HR means the possibility of tachycardia, and a low HR indicates sinus bradycardia dieses. [1]. So one of the most relevant tasks in automatic ECG analysis is the detection and characterization of every wave, particularly of the QRS complex, after which a more complete analysis can be obtained. Therefore, choosing the QRS detection algorithm is an essential step in the development of a real-time ECG analysis system [2].

Some quantitative performance comparisons of QRS detectors are presented in the literature [3], [4]. These evaluations are usually performed on a set of records extracted from standard ECG databases (such as the MIT-BIH, CSE, AHA,...) and are based on detection scores, generally expressed as sensitivity-specificity pairs. An average score, calculated over a set of different records, is assumed to reflect the overall performance of the detectors. A limitation of this method is that an ECG is composed of multiple noise levels and types, and a variety of beat morphologies.

In 2011, R. Harikumar and S.N. Shivappriya [5] investigated and compared a set of efficient techniques to extract and select striking features from the ECG data applicable in automatic cardiac beat classification. Each technique was applied to a pre-selected data segment from the MIT-BIH database. The classification and optimization of different heart beat methods were performed based upon the extracted features (morphological and statistical features). The morphological features were found as the most important for arrhythmia classification. However, because of ECG signal variability in different patients, the statistical approach is favoured for a precise and robust feature extraction. Among all those feature extraction, feature selection, classification and optimization techniques, SVM based PSO gave the higher classification accuracy with curse of dimensionality.

G. Jaswal, et al. [6] in 2012, used the Discrete Wavelet Transform (DWT) to detect QRS complex, and founded that, the DWT approach is the better and more accurate than the other common methods when evaluated on MIT/BIH ECG database. 
In this paper, a system for classification of the ECG signals is proposed. It consists of the following four main stages: ( $i$ ) a preprocessing stage for preparation; (ii) a stage analyzing the ECG signals using a QRS-like response FIR filter bank; (iii) a stage for feature selections and calculations; (iv) a stage for classifying cardiac diseases using a Fuzzy Inference System (FIS).

The rest of this paper is organized as follows: Section 2 explains the principles of ECG signal for some selected disease cases. The proposed system for the classification of ECG signals is presented in section 3 with the description of different stages. FIR filter efficient design method with its resulting significant coefficients and rules of the proposed FIS based classifier with the resulting accurate disease diagnosis are also illustrated in this section. Finally, section 4 concludes this paper.

\section{2- Principles of ECG Signal}

In the above section, properties of normal ECG signal is exhibited. This section presents ECG signals for the different disease cases adopted for this paper with the presentation of the healthy one. Such disease cases are shown in Figure 2 and described in the followings [7]:

\section{a. Left ventricular hypertrophy (LVH)}

$\mathrm{LVH}$ is the thickening of the myocardium (muscle) of the left ventricle of the heart [8]. ECG abnormalities that may be observed in patients with LVH are

- Increased QRS voltage .

- Secondary changes of the ST segment and/or T waves

- Left axis deviation (LAD): the electrical axis will tend to deviate to the left.

- Broadened QRS complex (more than $90 \mathrm{msec}$ ).

- ST segment depression and T wave inversion in the lateral leads [9].

\section{b. Hyperkalemia}

Hyperkalemia refers to the increase in the level of potassium in the blood. This case can be detected in the shape of ECG signal by the tall $\mathrm{T}$ wave and by flatting or absent $\mathrm{P}$ wave [10].

\section{c. Pacemaker}

The pacemaker is responsible for generating initial beat. Therefore, any problem in the pacemaker will make the $\mathrm{P}$ wave disappear [10].

\section{d. Ischaemic}

Ischaemic heart disease (IHD), or myocardial ischaemia is a disease characterized by ischaemia (reduced blood supply) of the heart muscle, usually due to coronary artery disease (atherosclerosis of the coronary arteries) [11]. ECG abnormalities that may be observed in patients with ischaemia are

- ST depression

- T wave inversion

- occasionally tall pointed $\mathrm{T}$ waves [12]. 


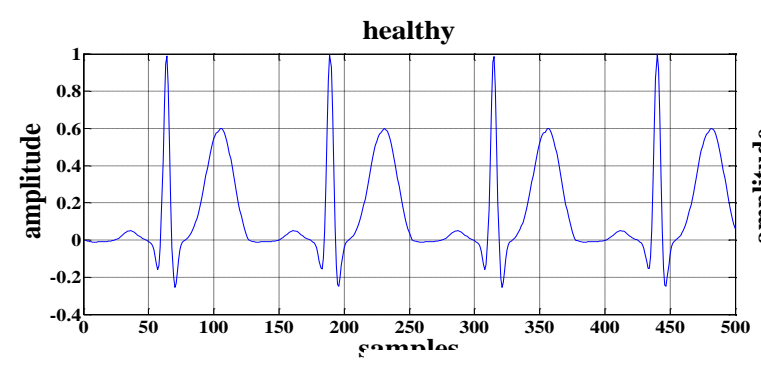

(a)

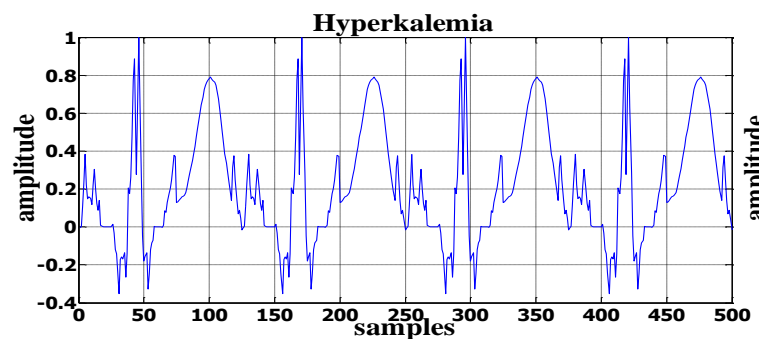

(c)

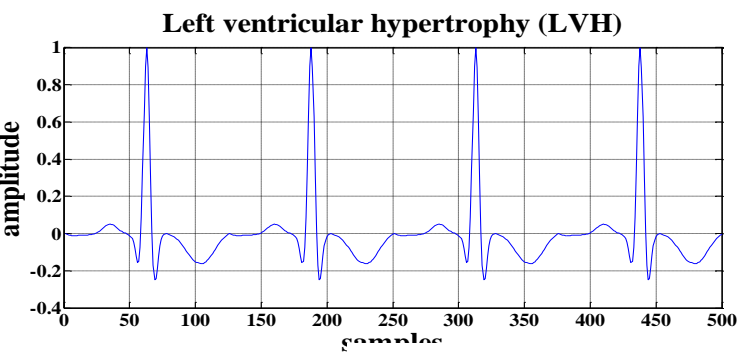

(b)

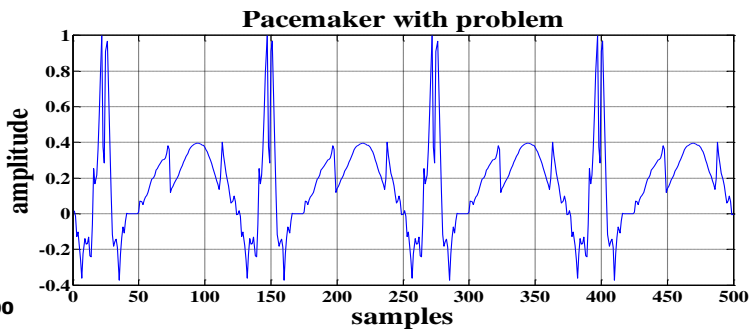

(d)

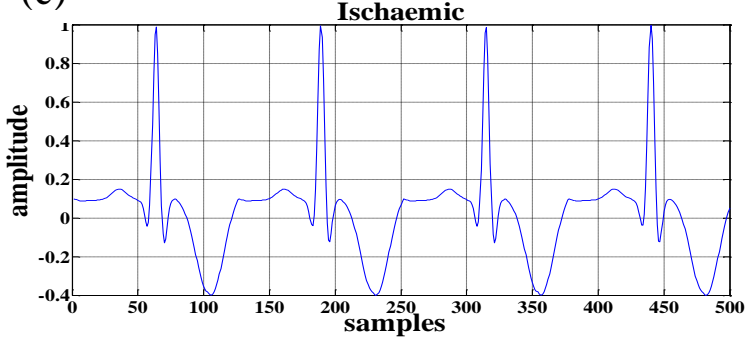

(e)

Fig. (2): ECG signal of a (a) healthy person (b) Left ventricular hypertrophy (LVH) (c) Hyperkalemia (d) Pacemaker with problem (e) Ischaemic.

\section{The Proposed System}

Block diagram of different stages of the system presented in this paper for classification of the ECG signals is shown in Figure 3. The main stages of such system is described in the following sub-sections. It should be noted that the preprocessing stage involves some amplitude and time scaling of the original ECG signal in addition to a proper sampling frame.

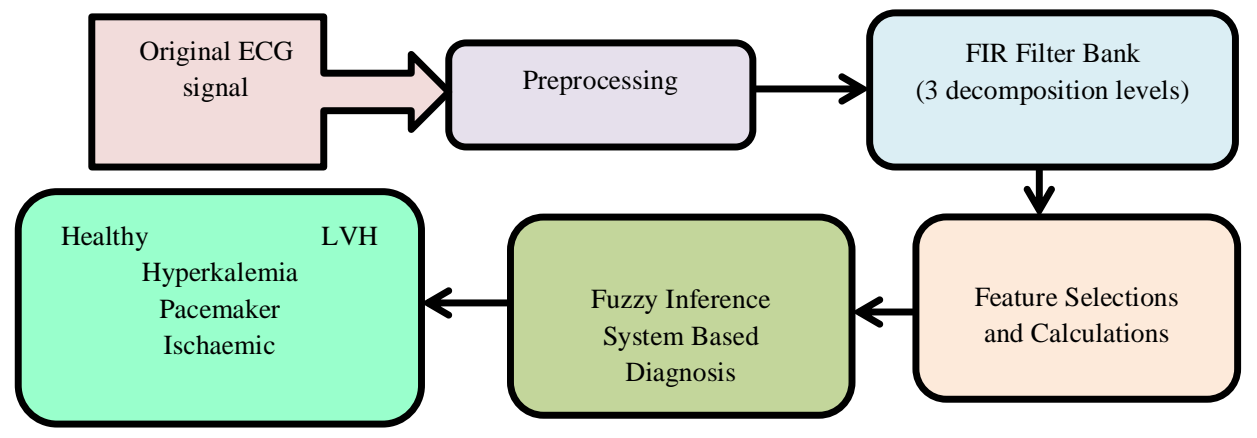

Fig. (3): Block diagram of the proposed classifier. 


\subsection{FIR filter bank}

In addition to the $\mathrm{P}, \mathrm{QRS} \& \mathrm{~T}$ waves in ECG signal, information related to cardiac diseases may also exist and can be extracted from the intervals and amplitudes of these waves. This paper proposes a system for detecting the QRS complex part. The first step of this system separates the ECG input signal into multiple coefficients using FIR filter bank. A QRS-like shape FIR filter bank is designed in order to extract as much information related to different cardiac diseases in QRS wave as possible. The $1^{\text {st }}$ order Gaussian derivative function has a similar shape to QRS complex part of the ECG and thus, can accurately utilized in the design of such filter bank for QRS feature extraction. Gaussian time function is defined by

$\psi(t, \sigma)=\frac{1}{\sigma \sqrt{2 \pi}} e^{-\left(\frac{(t-m)^{2}}{2 \sigma^{2}}\right)}$

where $\psi(t)$ is the Gaussian function in terms of the time $t, \sigma$ is the standard deviation and $m$ is the center of the wave (see Figure 4). The $1^{\text {st }}$ order Gaussian derivative function as in Figure 5 is defined by

$\psi^{\prime}(t, \sigma)=\frac{m-t}{\sigma^{3} \sqrt{2 \pi}} e^{-\left(\frac{(t-m)^{2}}{2 \sigma^{2}}\right)}$

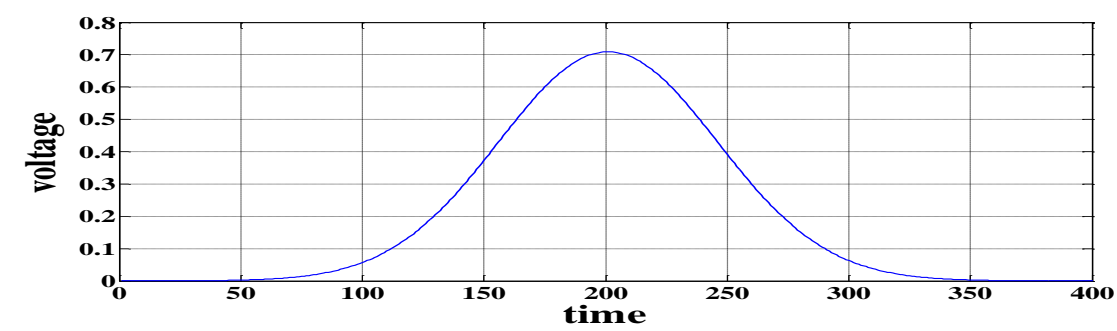

Fig. (4): The Gaussian function.

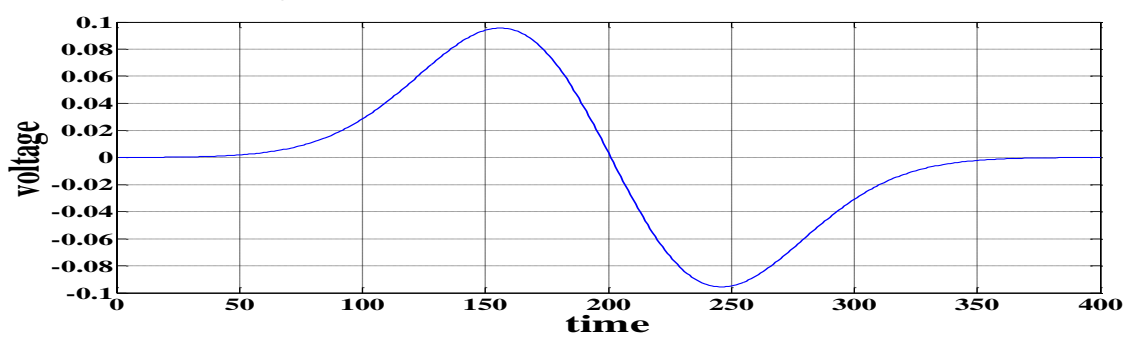

Fig. (5): The $1^{\text {st }}$ derivative Gaussian function.

The $1^{\text {st }}$ order derivative Gaussian system can be modeled using a Gaussian system convolution stage with a pre-differentiating stage of input signal $x(n)$ as shown in Figure 6 [13].

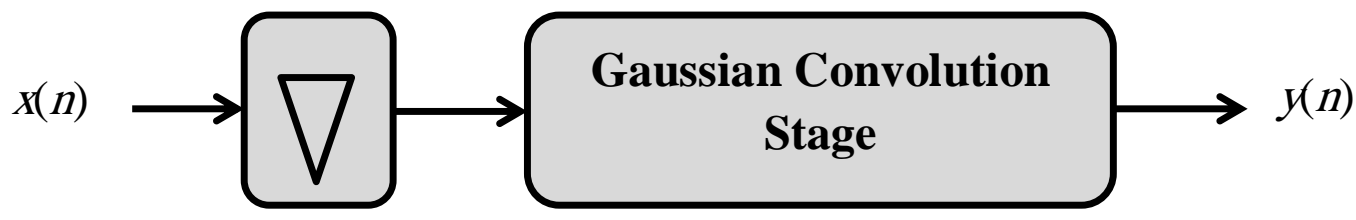

Fig. (6): A 1st order derivative Gaussian system model (using Gaussian function and a derivative stage).

The design of the corresponding digital FIR filter is accomplished by the determination of the values of such filter coefficients simulating the $1^{\text {st }}$ order Gaussian derivative. To 
determine the values of these coefficients, the value of the standard deviation $\sigma$ must first be assigned. The selected value of the standard deviation is $\sigma=4.5$, which is suitable to approximate the magnitude response of the designed FIR low-pass filter as a half band linear phase filter of the type shown in Figure 7. The corresponding impulse response for designed FIR low-pass filter is shown in Figure 8.

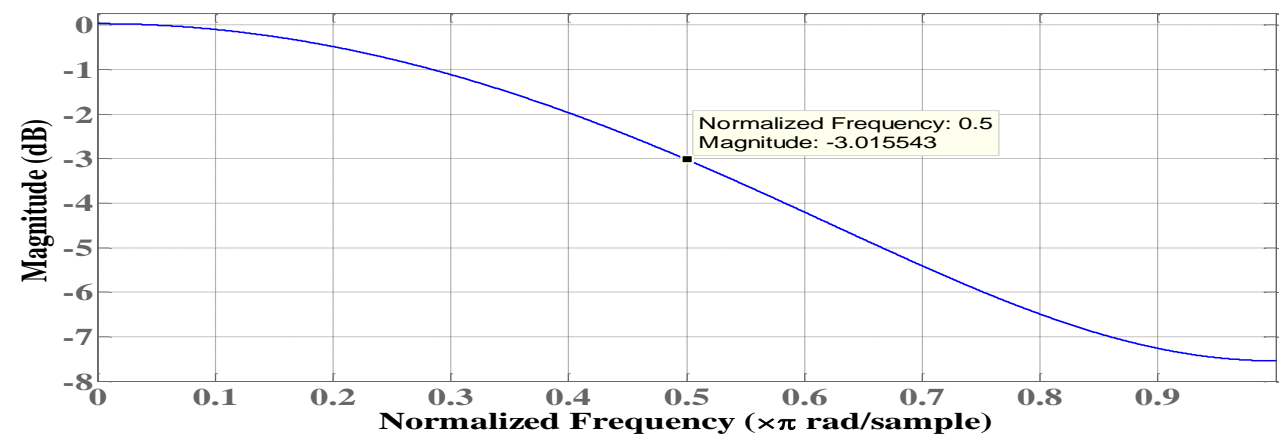

Fig. (7): Magnitude response for designed filter.

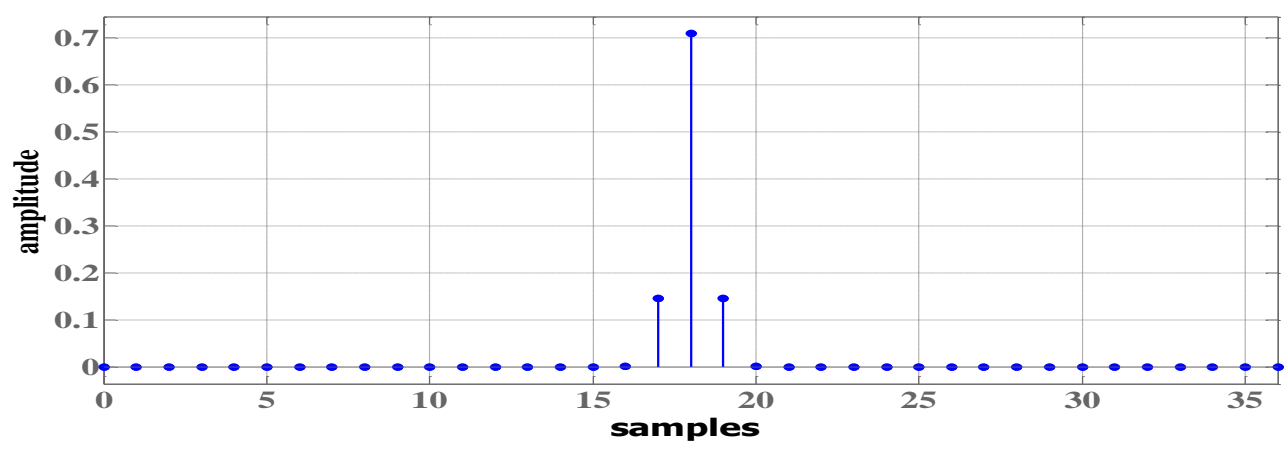

Fig. 8 Impulse response for designed filter.

From Figure 8, the filter impulse response contains 37 coefficients. The transfer function of filter is given by the following equation

$$
\mathrm{H}(\mathrm{z})=\mathrm{h}_{0}+\mathrm{h}_{1} \mathrm{z}^{-1}+\mathrm{h}_{2} z^{-2}+\mathrm{h}_{3} z^{-3}+\cdots+\mathrm{h}_{34} z^{-34}+\mathrm{h}_{35} z^{-35}+\mathrm{h}_{36} \mathrm{z}^{-36}
$$

The coefficients values are given inTable1.

Table (1): The coefficients values of the designed filter.

\begin{tabular}{|c|c|c|c|}
\hline Coefficient & The value & Coefficient & The value \\
\hline $\mathrm{h}_{0}=\mathrm{h}_{36}$ & $3.105 \times 10^{-223}$ & $\mathrm{~h}_{10}=\mathrm{h}_{26}$ & $8.474 \times 10^{-45}$ \\
\hline $\mathrm{h}_{1}=\mathrm{h}_{35}$ & $3.253 \times 10^{-199}$ & $\mathrm{~h}_{11}=\mathrm{h}_{25}$ & $1.669 \times 10^{-34}$ \\
\hline $\mathrm{h}_{2}=\mathrm{h}_{34}$ & $1.445 \times 10^{-176}$ & $\mathrm{~h}_{12}=\mathrm{h}_{24}$ & $1.394 \times 10^{-25}$ \\
\hline $\mathrm{h}_{3}=\mathrm{h}_{33}$ & $2.723 \times 10^{-155}$ & $\mathrm{~h}_{13}=\mathrm{h}_{23}$ & $4.937 \times 10^{-18}$ \\
\hline $\mathrm{h}_{4}=\mathrm{h}_{32}$ & $2.175 \times 10^{-135}$ & $\mathrm{~h}_{14}=\mathrm{h}_{22}$ & $7.415 \times 10^{-12}$ \\
\hline $\mathrm{h}_{5}=\mathrm{h}_{31}$ & $7.368 \times 10^{-117}$ & $\mathrm{~h}_{15}=\mathrm{h}_{21}$ & $4.722 \times 10^{-7}$ \\
\hline $\mathrm{h}_{6}=\mathrm{h}_{30}$ & $1.058 \times 10^{-99}$ & $\mathrm{~h}_{16}=\mathrm{h}_{20}$ & 0.001275 \\
\hline $\mathrm{h}_{7}=\mathrm{h}_{29}$ & $6.448 \times 10^{-84}$ & $\mathrm{~h}_{17}=\mathrm{h}_{19}$ & 0.146 \\
\cline { 1 - 2 } $\mathrm{h}_{8}=\mathrm{h}_{28}$ & $1.665 \times 10^{-69}$ & $\mathrm{~h}_{18}$ & \multirow{2}{*}{0.7092} \\
\hline $\mathrm{h}_{9}=\mathrm{h}_{27}$ & $1.824 \times 10^{-56}$ & & \multicolumn{2}{|c}{} \\
\cline { 1 - 2 }
\end{tabular}


From Table 1, it can be seen that most of the filter coefficients are of very small values and can thus be neglected. The designed filter length will be truncated to 5 coefficients only. The resulting transfer function is

$$
\mathrm{H}(\mathrm{z})=\mathrm{h}_{16} \mathrm{z}^{-16}+\mathrm{h}_{17} \mathrm{z}^{-17}+\mathrm{h}_{18} z^{-18}+\mathrm{h}_{19} z^{-19}+\mathrm{h}_{20} z^{-20}
$$

Multiplying (4) by $\mathrm{z}^{16}$, another final causal truncated low-pass filter function with less delay can be rewritten as

$$
\mathrm{H}(z)=\mathrm{h}_{16}+\mathrm{h}_{17} \mathrm{z}^{-1}+\mathrm{h}_{18} z^{-2}+\mathrm{h}_{19} z^{-3}+\mathrm{h}_{20} z^{-4}
$$

The magnitude response of this final designed filter with the causal truncated transfer function is shown in Figure 9.

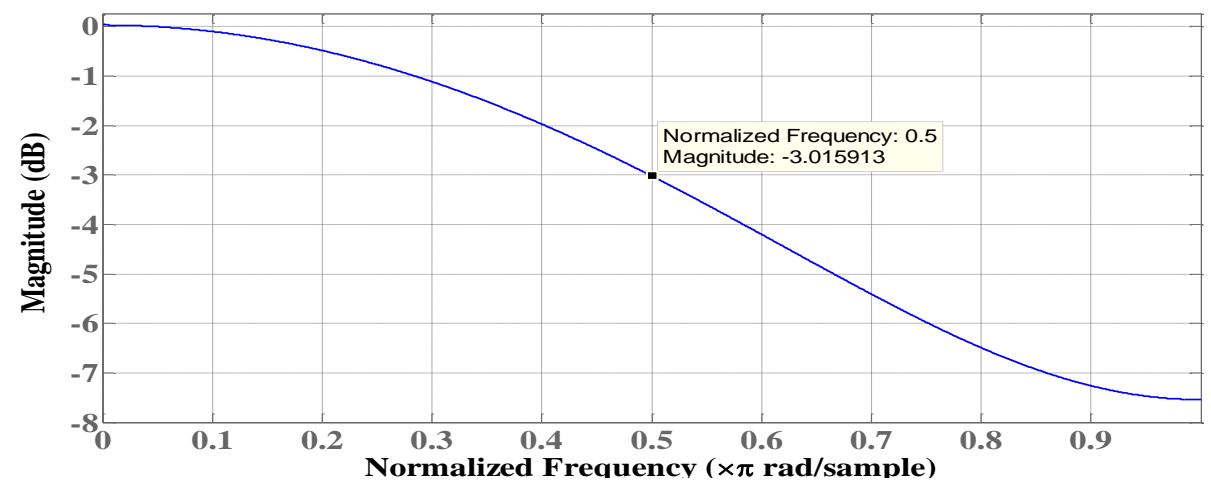

Fig. (9): Magnitude response for designed filter with the truncated transfer function.

By the property of quadrature mirror filters (QMFs), $\mathrm{G}(\mathrm{z})=\mathrm{H}(-\mathrm{z})$, the high-pass system function $\mathrm{G}(\mathrm{z})$ can be written as

$$
\mathrm{G}(z)=\mathrm{h}_{16}-\mathrm{h}_{17} \mathrm{z}^{-1}+\mathrm{h}_{18} z^{-2}-\mathrm{h}_{19} z^{-3}+\mathrm{h}_{20} z^{-4}
$$

The filter bank polyphase representations are expressed as

$$
\begin{aligned}
& \quad \mathrm{H}_{\text {even }}\left(z^{2}\right) \\
& =\mathrm{h}_{16}+\mathrm{h}_{18} z^{-2}+\mathrm{h}_{20} z^{-4} \\
& \text { and } \\
& \begin{array}{r}
\mathrm{H}_{\text {odd }}\left(z^{2}\right)=\mathrm{h}_{17} \\
\qquad \mathrm{~h}_{19} z^{-2}
\end{array}
\end{aligned}
$$

When a pre-down sampling (by 2) stage is introduced before each of the two filter bank branches, the even and odd transfer functions $\left[\mathrm{H}_{\text {even }}\left(z^{2}\right)\right.$ and $\mathrm{H}_{o d d}\left(z^{2}\right)$, in equations (7) and (8), respectively] can be rewritten in terms of $z$, as

$$
\begin{aligned}
& \mathrm{H}_{\text {even }}(z)=\mathrm{h}_{16}+\mathrm{h}_{18} z^{-1}++\mathrm{h}_{20} z^{-2} \\
& \text { and } \\
& \mathrm{H}_{\text {odd }}(z)=\mathrm{h}_{17}+\mathrm{h}_{19} z^{-1}
\end{aligned}
$$

The low-pass transfer function in (5) and high-pass one in (6) can then be reduced, respectively to

$$
\begin{aligned}
& \mathrm{H}(z)=\mathrm{H}_{\text {even }}(z)+z^{-1} \mathrm{H}_{\text {odd }}(z) \\
& \text { and } \\
& \mathrm{G}(z)=\mathrm{H}_{\text {even }}(z)-z^{-1} \mathrm{H}_{\text {odd }}(z)
\end{aligned}
$$

The lattice structure for the designed final FIR filter bank is shown in Figure 10. 


\section{Al-Rafidain Engineering

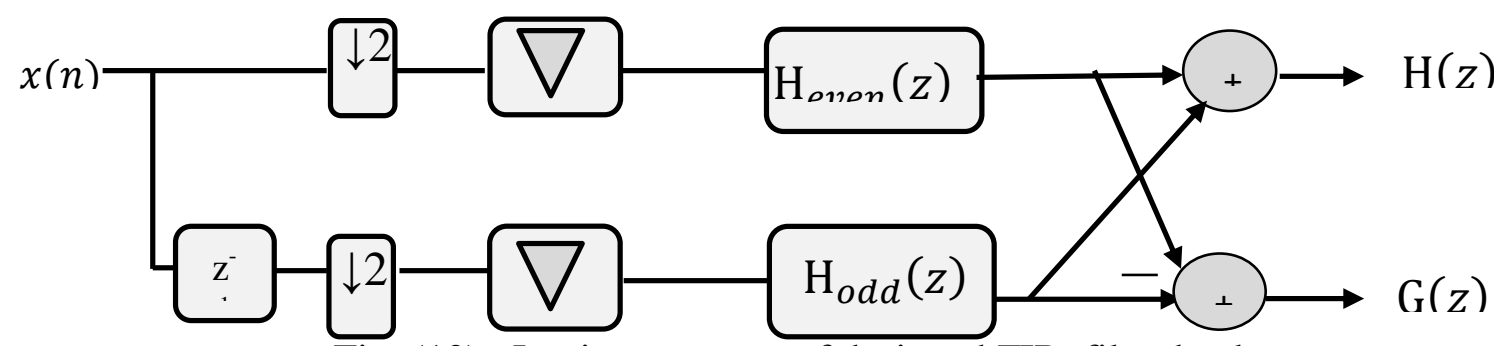

Fig. (10): Lattice structure of designed FIR filter bank.

\subsection{Feature selection}

The energy distributions for 8 signals obtained from the 3-level decompositions of the designed filter bank (LLL, LLH, LHL, LHH, HLL, HLH, HHL, HHH) are shown in Figure 11 for different disease classes. The statistical variance values are calculated for all energy values and then used as inputs to the FIS stage. These variance values shown in Figure 12.

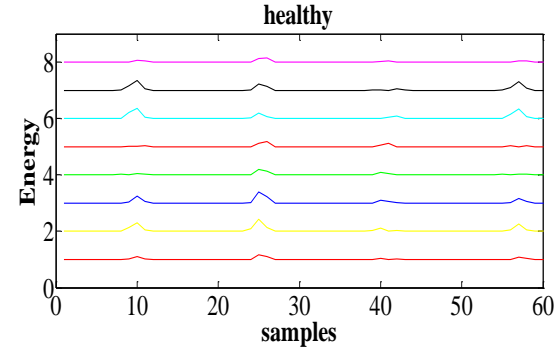

(a)

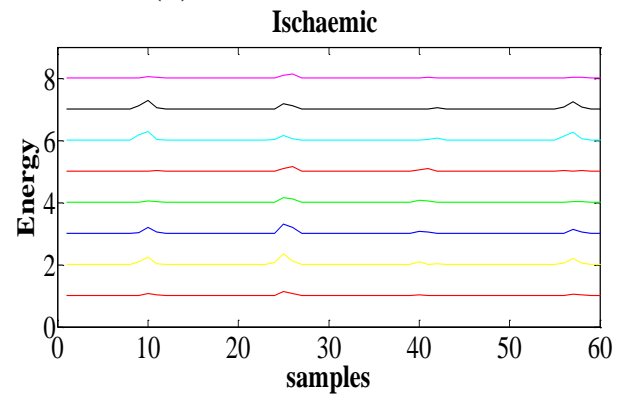

(d)

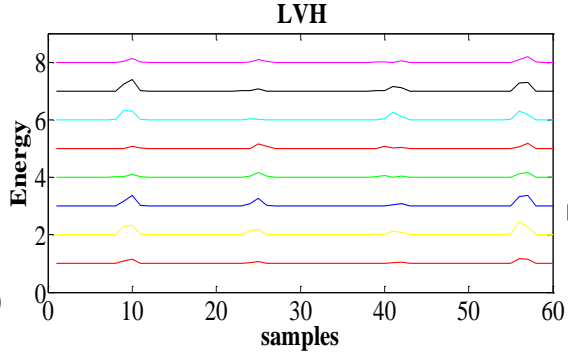

(b)

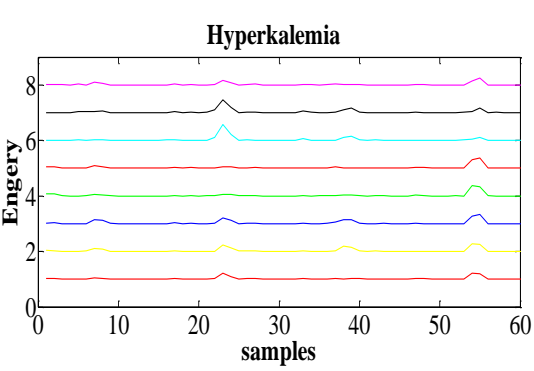

(c)

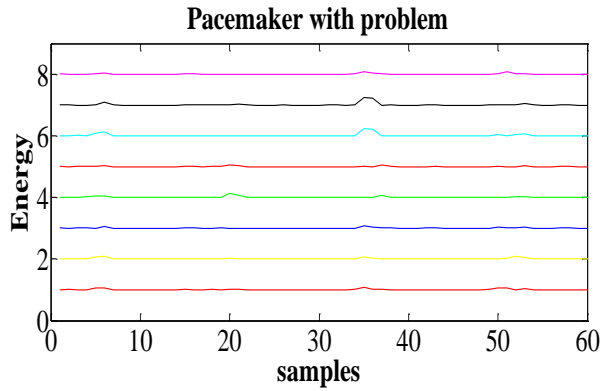

(e)

Fig. (11): Waveforms of various energy values for each ciass; (a) healthy person

(b) LVH (c) Hyperkalemia (d) Pacemaker with problem (e) Ischaemic.

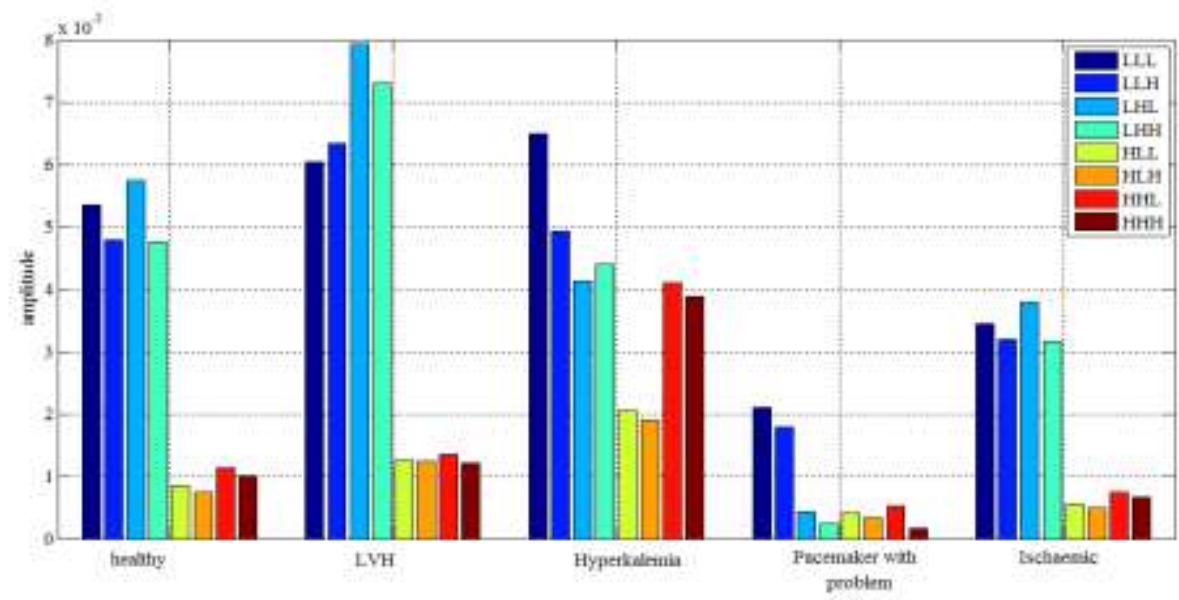

Fig. (12) Variances of energy values for different disease cases. 


\subsection{Fuzzy inference system based diagnosis}

In this sub-section, a fuzzy logic for the diagnosis of the cardiac diseases is introduced. A Fuzzy Inference System (FIS) is developed in this paper using the Fuzzy Logic Toolbox graphical user interface (GUI) [14]. Such FIS is shown in Figure 13, and is composed of the followings:

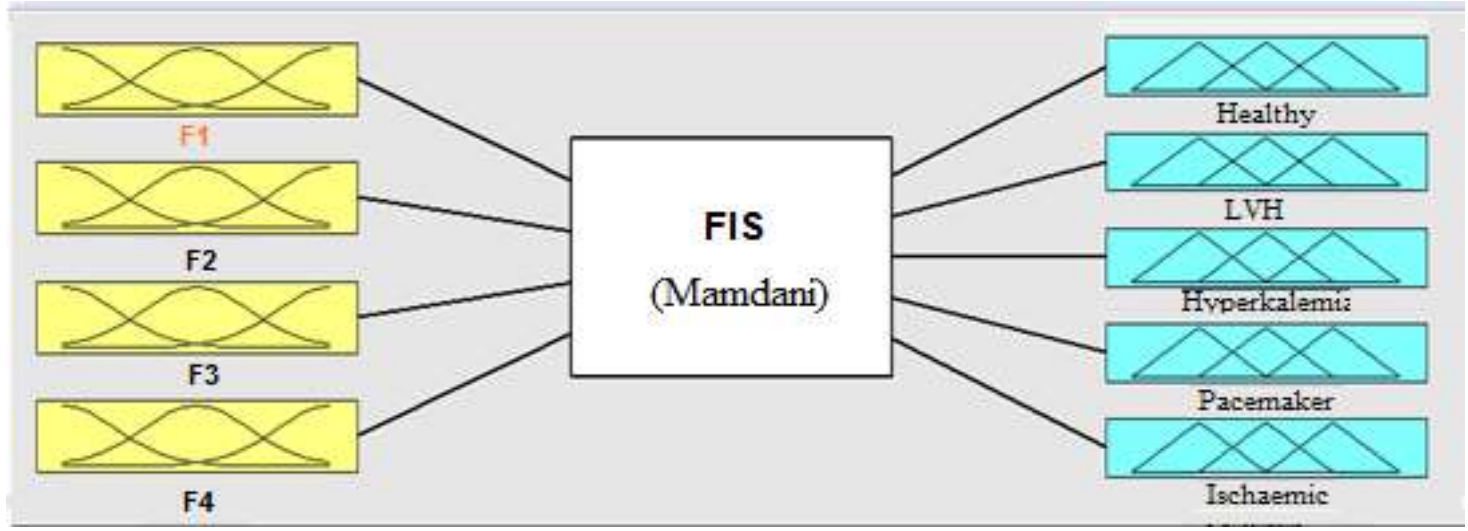

Fig. (13) The FIS structure for the ECG classifier.

- Four input membership descriptors representing the feature space; F1, F2, F3, and F4, indicating only the significant variance values for LHL, LHH, LLL, and HHH signals, respectively. Each of these inputs has five cases ("very_low", "low, "medium", "high", and "very_high"), as shown in Figure 14.

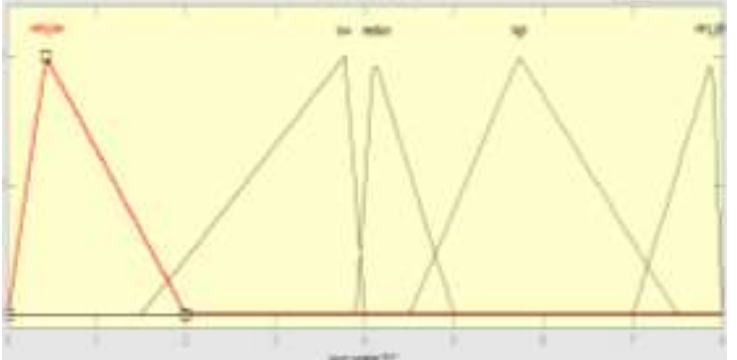

(a)

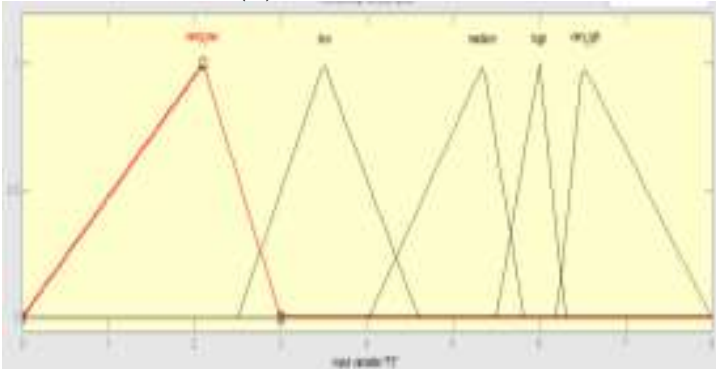

(c)

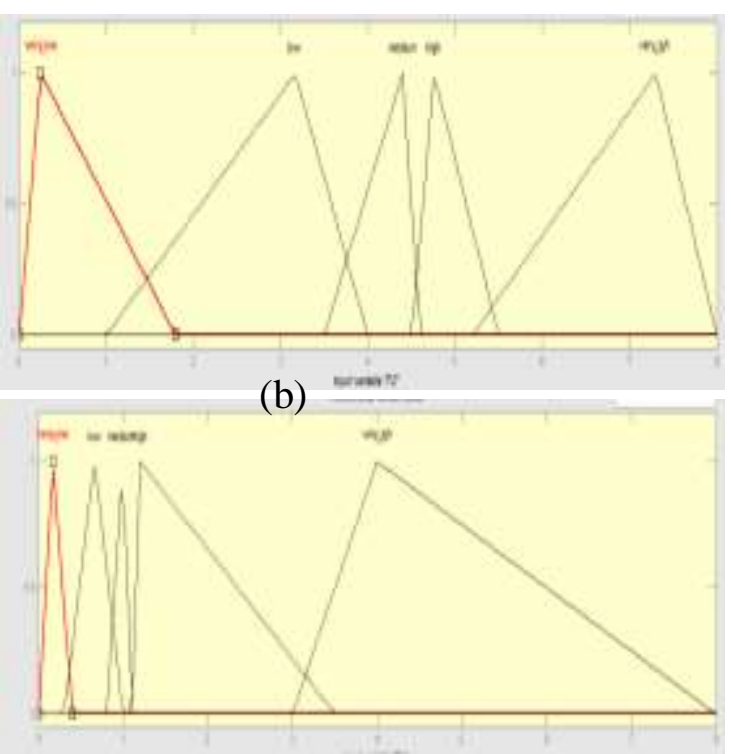

(d)

Fig. (14): Input membership functions; (a) F1, (b) F2, (c) F3, and (d) F4.

- Five output membership descriptors for the diseased cases ( Healthy, LVH, Hyperkalemia, Pacemaker with problem, and Ischaemic), and have three cases ("Un-likely", "Likely", and "Highly-likely"). The output membership function for the class "Healthy" shown in Figure 15. Other classes are also carry the same in structures. 


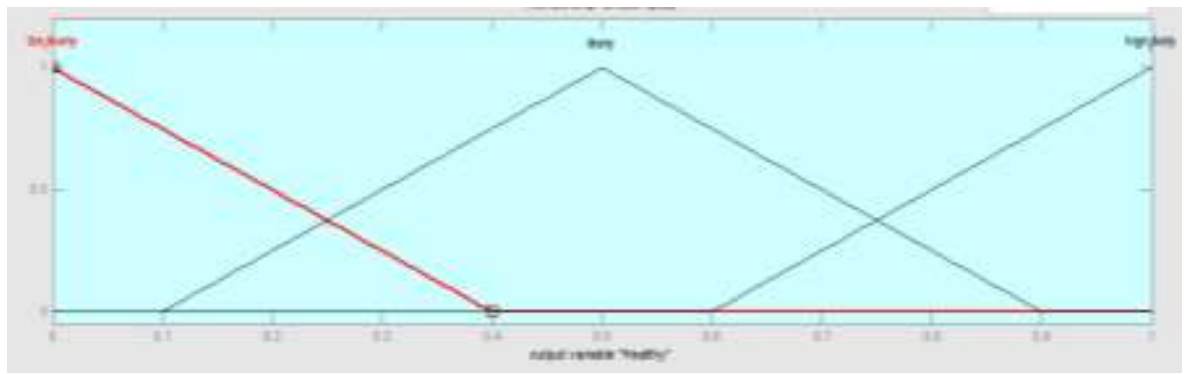

Fig. (15): Healthy membership functions.

- A set of 6 rules for the diagnosis of the cardiac diseases can be constructed as shown in Table 2. Based on the intersection percentages, the values are allocated and a total decision boundary (surface) is calculated whose center is the De-fuzzified output for the system.

Table (2): Rule base of ECG classifier.

\begin{tabular}{|c|c|c|c|c|c|c|c|c|c|}
\hline Rules & F1 & F2 & F3 & F4 & Healthy & LVH & $\begin{array}{c}\text { Hyperkale } \\
\text { mia }\end{array}$ & $\begin{array}{c}\text { Pacemaker } \\
\text { with problem }\end{array}$ & Ischaemic \\
\hline 1 & - & - & $\begin{array}{c}\text { very } \\
\text { low }\end{array}$ & $\begin{array}{c}\text { very } \\
\text { low }\end{array}$ & Un-likely & $\begin{array}{c}\text { Un- } \\
\text { likely }\end{array}$ & Un-likely & Highly-likely & Un-likely \\
\hline 2 & - & - & low & low & Un-likely & $\begin{array}{c}\text { Un- } \\
\text { likely }\end{array}$ & Un-likely & Un-likely & Highly-likely \\
\hline 4 & high & high & medium & - & $\begin{array}{c}\text { Highly- } \\
\text { likely }\end{array}$ & $\begin{array}{c}\text { Un- } \\
\text { likely }\end{array}$ & Un-likely & Un-likely & Un-likely \\
\hline 5 & medium & medium & $\begin{array}{c}\text { very } \\
\text { high }\end{array}$ & $\begin{array}{c}\text { very } \\
\text { high }\end{array}$ & Un-likely & $\begin{array}{c}\text { Un- } \\
\text { likely }\end{array}$ & $\begin{array}{c}\text { Highly- } \\
\text { likely }\end{array}$ & Un-likely & Un-likely \\
\hline 6 & medium & medium & medium & $\begin{array}{c}\text { mediu } \\
\mathrm{m}\end{array}$ & Likely & $\begin{array}{c}\text { Un- } \\
\text { likely }\end{array}$ & Likely & Un-likely & Un-likely \\
\hline
\end{tabular}

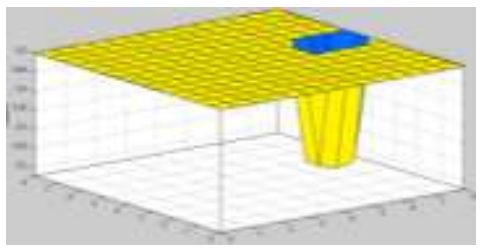

(a)

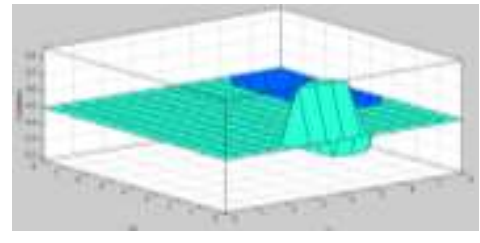

(b)

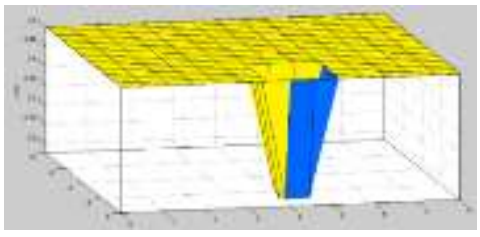

(c)

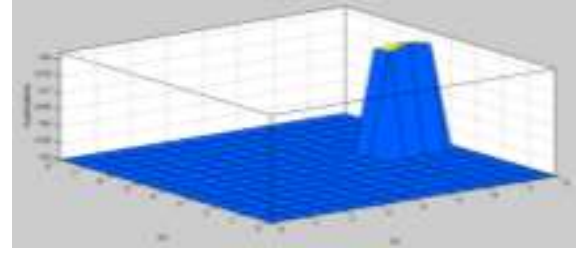

(d)

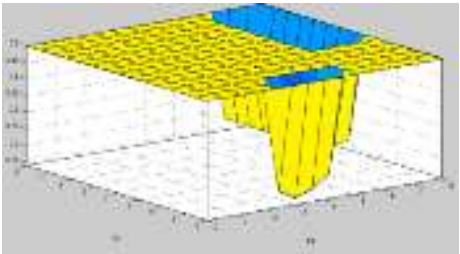

(e)

Fig. (16): Surfaces for (a) Healthy, between "F3" and "F2"(b) LVH, between "F3" and "F1" (c) Hyperkalemia, between "F3" and "F2" (d) Pacemaker, between "F3" and "F4", and (e) Ischaemic, between "F3" and "F4". 
The resulting surfaces are multidimensional and cannot be exposed as one hyper surface. However, some subclass surfaces can be plotted and are shown in Figure 16. The classified results are shown in Table 3. These results indicate almost $100 \%$ accurate detections for the five tested diseased cases. Other cases can be likewise incorporated in the rule base and later into the whole classification system.

Table (3): Results of the ECG classifier.

\begin{tabular}{|c|c|c|c|c|c|c|c|c|}
\hline F1 & F2 & F3 & F4 & Healthy & LVH & Hyperkalemia & $\begin{array}{c}\text { Pacemaker } \\
\text { with } \\
\text { problem }\end{array}$ & Ischaemic \\
\hline 5.7 & 4.7 & 5.3 & 1 & 0.8614 & 0.1386 & 0.1386 & 0.1386 & 0.1386 \\
\hline 0.45 & 0.25 & 2.1 & 0.1 & 0.13 & 0.13 & 0.13 & 0.87 & 0.13 \\
\hline 3.8 & 3.16 & 3.4 & 0.7 & 0.1352 & 0.1352 & 0.1352 & 0.1352 & 0.8648 \\
\hline 7.9 & 7.3 & 6 & 1.2 & 0.13 & 0.87 & 0.13 & 0.13 & 0.13 \\
\hline 4.1 & 4.4 & 6.4 & 3.88 & 0.1468 & 0.1468 & 0.8532 & 0.1468 & 0.1468 \\
\hline
\end{tabular}

\section{Conclusions}

In this paper, a less-complex FIR filter bank has been efficiently designed using lattice structures for QRS features extraction. A $1^{\text {st }}$ order Gaussian derivative function which simulates the shape of the QRS complex part of the ECG signal has been utilized in such design. ECG features have been taken after three-level decompositions of the proposed filter bank. Significant energy values of the filter bank output coefficients have been calculated for five different tested disease cases. Such values are treated as crucial points for the design of a rule-based fuzzy classifier. Almost 100\% accurate detections for the five tested diseased cases have been obtained.

\section{References}

[1] J. Pan and W. J. Tompkins, "A real-time QRS detection algorithm," IEEE Trans. Biomed. Eng., vol. BME-32, No. 3, pp. 230-236, Mar.1985.

[2] G. Carrault, M. O. Cordier, R. Quiniou and F. Wang, 'Temporal abstraction and inductive logic programming for arrhythmia recognition from electrocardiograms', Artificial Intelligence in Medicine Vol. 28, Issue 3, pp. 231-263, July 2003.

[3] S. Kadambe, R. Murray, and F. Boudreaux-Bartels, "Wavelet transform-based QRS complex detector', IEEE Trans. Biomed. Eng., Vol. 46, Issue 7, pp. 838-848, July 1999.

[4] D. Benitez, P. Gaydecki, A. Zaidi, and A. Fitzpatrick, " The use of the Hilbert transform in ECG signal analysis', Comput. Biol. Med., Vol. 31 , Issue 5 , pp. 399-406, September 2001.

[5] R. Harikumar and S. N. Shivappriya, "Analysis of QRS Detection Algorithm for Cardiac Abnormalities-A Review", International Journal of Soft Computing and Engineering (IJSCE), ISSN: 2231-2307, Vol. 1, Issue 5, November 2011.

[6] G. Jaswal, R. Parmar, and A. Kaul, "QRS Detection Using Wavelet Transform", International Journal of Engineering and Advanced Technology (IJEAT) ISSN: 22498958, Vol. 1, Issue 6, pp. 1-5, August 2012.

[7] The MIT-BIH $\quad$ Database. $\quad$ On http://physionet.ph.biu.ac.il/physiobank/database/ 
[8] "Left ventricular hypertrophy (LVH)", from EKG interpretation On http://www.ekginterpretation.com/library/left-ventricular-hypertrophy-lvh

[9] "Left Ventricular Hypertrophy-LVH", from doktorekg.com $\quad$ On http://www.metealpaslan.com/ecg/lvhen.htm

[10] N. A. Boon ,N. R. colledge, B. R. walker, "Davidson principle and practical of medicine", churchil livingstone, twentieth, 2006.

[11] "Ischaemic heart disease", from Wikipedia, the free encyclopedia. On https://en.wikipedia.org/wiki/Ischaemic_heart_disease

[12] "ECG changes in ischaemic myocardium", from GPnotebook. On https://www.gpnotebook.co.uk/simplepage.cfm?ID=214958100

[13] Jassim M. Abdul-Jabbar and Abdulhamed M. Jasim, "Design and MultiPlierless Realization of ECG-Based Gaussian Wavelet Filter with Lattice Structures", Al-Rafidain Engineering Journal, Vol. 21, No. 2, April 2013.

[14] "Fuzzy Logic Toolbox", On http://www.mathworks.com/products/fuzzylogic/ 